\section{Temperature-compensated CMOS ring oscillator for power-management circuits}

K.N. Leung, C.H. Lo, P.K.T. Mok, Y.Y. Mai, W.Y. Leung and M.J. Chan

The design of a temperature-compensated CMOS ring oscillator is introduced. The concept is to make use of a linear, positively or negatively temperature-sloped supply voltage to power-up the ring oscillator. Experimental results show that the oscillation frequency remains fairly constant using the proposed scheme.

Introduction: Supply-and temperature-independent switching frequency $\left(f_{o s c}\right)$ is important in the design of switched-mode regulators for power management since this avoids many problems, such as frequency shifting of sub-harmonics in the frequency spectrum. In many commercial products, external low-tempco capacitors and resistors are used for generating the adjustable and low-tempco $f_{\text {osc }}$. However, this approach increases the cost of packaging owing to the extra pins, and it impacts the board size in compact applications. It has been shown by many publications that the temperature effects of mobility of carriers and the threshold voltage of MOSFET can be mutually cancelled, to generate a temperatureindependent voltage $[1,2]$. Based on this concept, a temperature-independent current $\left(I_{\text {osc }}\right)$ can be created similarly, such that the charging and discharging currents are constant. Since $f_{o s c} \propto 1 / I_{o s c}, f_{o s c}$ is a constant as well. The supply voltage is certainly a key parameter to achieve constant $I_{o s c}$, in addition to the size of the transistors.

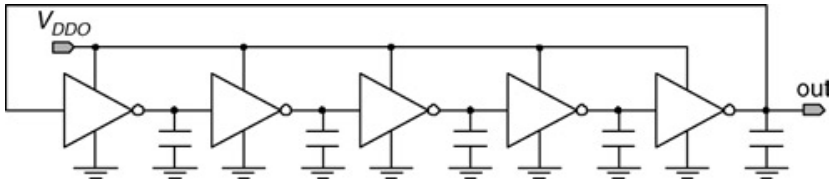

Fig. 1 Five-stage ring oscillator
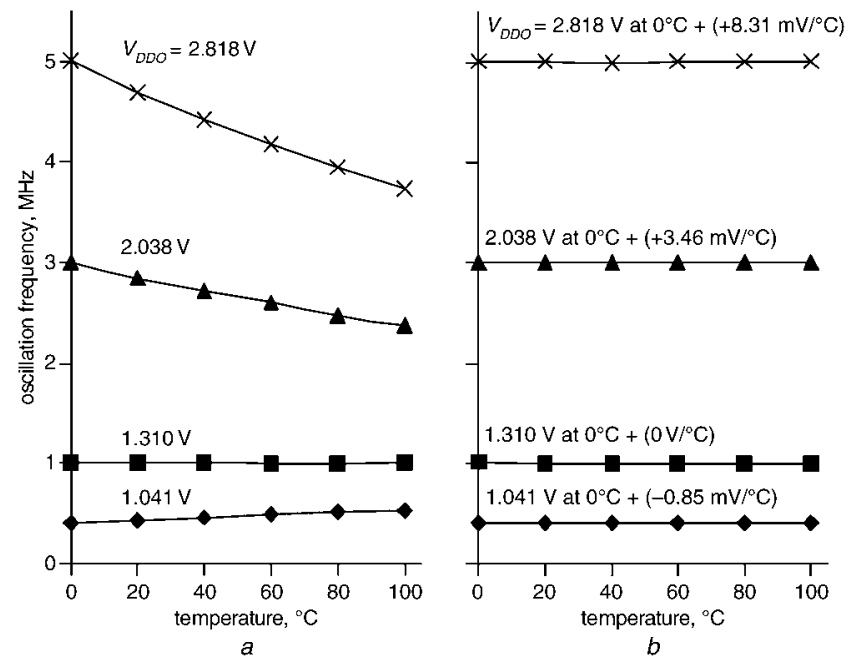

Fig. 2 Measured oscillation frequency at different temperatures with constant supply voltage and with temperature-gradient supply voltage

$a$ Constant supply voltage

$b$ Temperature-gradient supply voltage

Analysis of ring oscillator: A five-stage ring oscillator, shown in Fig. 1, has been implemented in $0.35 \mu \mathrm{m}$ CMOS technology. Fig. $2 a$ shows $f_{\text {osc }}$ against temperature and shows large variation of $f_{\text {osc }}$. When $V_{D D O}$ is high $\left(V_{D D O}=2.818 \mathrm{~V}\right.$ and $\left.2.038 \mathrm{~V}\right), f_{O S C}$ decreases with temperature. When $V_{D D O}$ is low $\left(V_{D D O}=1.041 \mathrm{~V}\right), f_{o s c}$ increases with temperature. However, at a particular $V_{D D O}\left(V_{D D O}=1.310 \mathrm{~V}\right)$, $f_{\text {osc }}$ can be kept constant. This reveals a fact that a constant $f_{\text {osc }}$ can be achieved by adjusting $V_{D D O}$. Based on the study, $V_{D D O}$ is designed to be linearly dependent of temperature. The temperature slope of $V_{D D O}$ can be positive or negative, which depends on the required magnitude of $V_{D D O}$ for the required $f_{O S C}$ in the applications. A range of about $0.3-5 \mathrm{MHz}$, which is the typical range of the switching frequency of the regulators, is chosen in this study. From Fig. $2 b$, it is found that $f_{\text {osc }}$ can be kept fairly constant by this approach. The required temperature slope is not large, so that $V_{D D O}$ is still within the bound of the maximum supply of typical IC technologies.

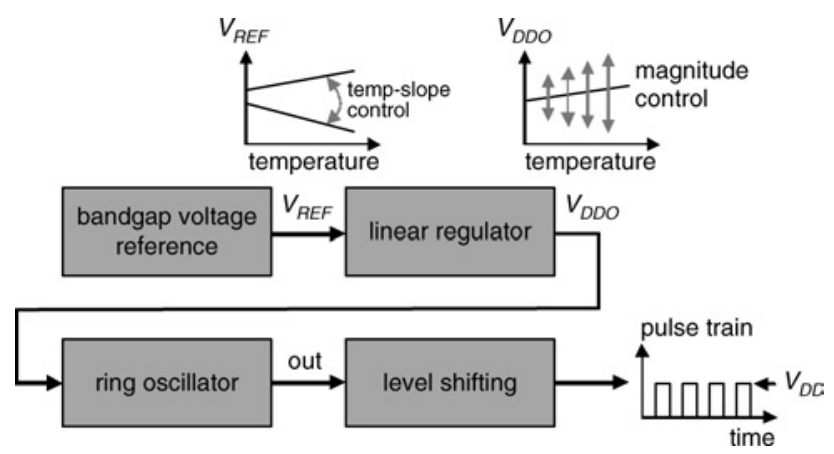

Fig. 3 Proposed approach

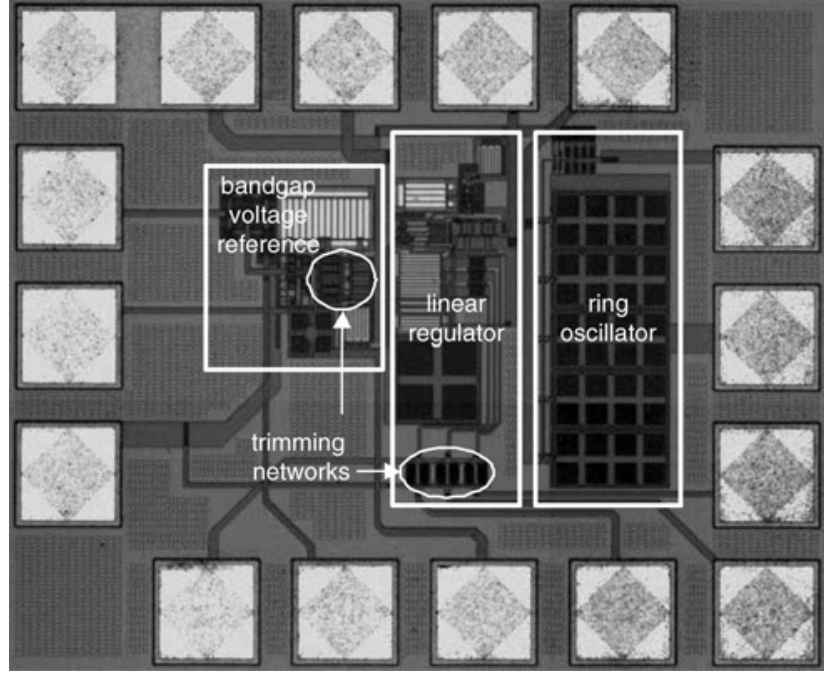

Fig. 4 Micrograph

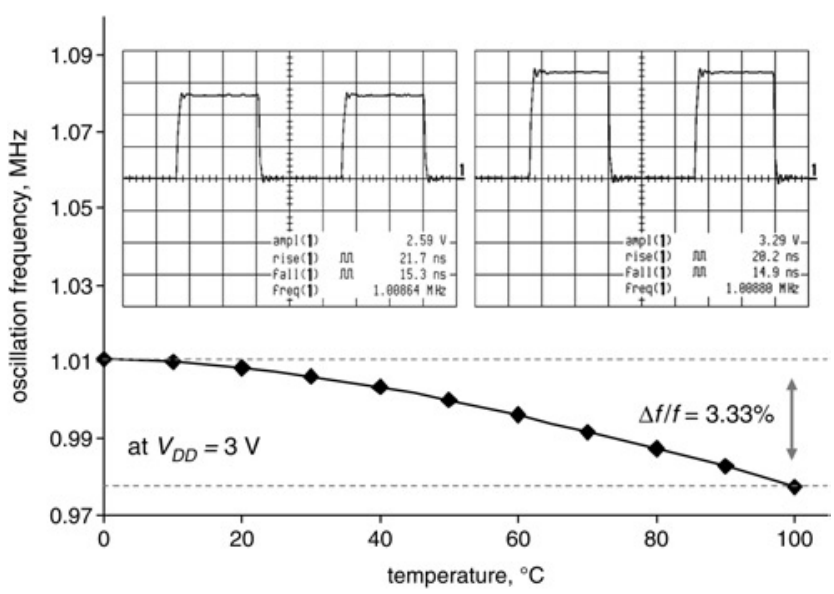

Fig. 5 Measured oscillation frequency with proposed method and measured pulse trains

Method: The proposed method implemented by a first-order bandgap voltage reference [3] and a linear regulator is shown in Fig. 3. The temperature slope of the reference voltage $\left(V_{R E F}\right)$ can be adjusted by resistance ratio [3]. Either a positive or a negative linear (approximated) temperature slope can be generated. $V_{R E F}$ can be scaled by the linear regulator to generate $V_{D D O}$. As a result, the ring oscillator is powered by $V_{D D O}$, which is temperature-sloped and magnitude adjustable. Therefore, $V_{D D O}$ is given by

$$
V_{D D O}=k V_{R E F}
$$

where $k$ is a temperature-independent constant, which is defined by the feedback resistors of the linear regulator [3]. From (1), the temperature dependence can be obtained as 


$$
\frac{\partial V_{D D O}}{\partial T}=k \frac{\partial V_{R E F}}{\partial T}
$$

Equation (2) shows that the temperature slope of $V_{D D O}$ can be adjusted by both $k$ and the temperature slope of $V_{R E F}$. The output voltage level of the ring oscillator can simply be restored by a level-shifting circuit. Therefore, the amplitude of the pulse train of the ring oscillator is $V_{D D}$, but $f_{o s c}$ is $V_{D D}$-independent.

Experimental results: The proposed approach has been implemented in silicon. Fig. 4 shows a micrograph, in which each building block is indicated. The active chip area is about $450 \times 360 \mu \mathrm{m}$, excluding the test pads. Fig. 5 shows a test case that targets $f_{o s c}=1 \mathrm{MHz}$ at $V_{D D}=3 \mathrm{~V}$. The error is $3.33 \%$ in the temperature range $0-100^{\circ} \mathrm{C}$. The accuracy is sufficient for the typical switched-mode regulator design. Fig. 5 also shows a capture of the output waveforms. The ring oscillator is supplied by temperature-dependent $V_{D D O}$, while $V_{D D}$ is tested at room temperature at about 2.6 and $3.3 \mathrm{~V}$. The amplitudes of the pulse train in the two cases are therefore 2.6 and $3.3 \mathrm{~V}$, while the change of $f_{\text {osc }}$ is negligible. This proves that the supply is independent of the proposed implementation.

Conclusions: An approach to power-up a ring oscillator has been introduced, discussed and proved experimentally, by introducing a temperature-sloped and magnitude-adjustable supply voltage. The approach is simple and is helpful in regulator design to eliminate external components.
Acknowledgement: This work was supported by the Research Grant Council of Hong Kong SAR Government under project number HKUST 617705.

(C) The Institution of Engineering and Technology 2007

2 April 2007

Electronics Letters online no: 20070935

doi: 10.1049/el:20070935

K.N. Leung (Department of Electronic Engineering, The Chinese University of Hong Kong, Shatin, Hong Kong, People's Republic of China)

C.H. Lo, P.K.T. Mok, Y.Y. Mai, W.Y. Leung and M.J. Chan (Department of Electronic and Computer Engineering, The Hong Kong University of Science and Technology, Clear Water Bay, Hong Kong, People's Republic of China)

E-mail: eemok@ee.ust.hk

\section{References}

1 Manku, T., and Wang, Y.: 'Temperature-independent output voltage generated by threshold voltage of an NMOS transistor', Electron. Lett., 1995, 31, (12), pp. 935-936

2 Fiori, F., and Crovetti, P.S.: 'Compact temperature compensated CMOS current reference', Electron. Lett., 2003, 39, (1), pp. 42-43

3 Gray, P.R., Hurst, P.H., Lewis, S.H., and Meyer, R.G.: 'Analysis and design of analog integrated circuits' (Wiley, New York, 2001, 4th edn.) 